\title{
The Role of the Apelin/APJ System in the Regulation of Liver Disease
}

\author{
Xinrui Lv' ${ }^{1}$, Jing Kong ${ }^{1}$, Wei-Dong Chen ${ }^{1,2 *}$ and Yan-Dong Wang ${ }^{3 *}$ \\ 1 Key Laboratory of Receptors-Mediated Gene Regulation and Drug Discovery, School of Medicine, Henan University, \\ Kaifeng, China, ${ }^{2}$ Key Laboratory of Molecular Pathology, School of Basic Medical Science, Inner Mongolia Medical \\ University, Hohhot, China, ${ }^{3}$ State Key Laboratory of Chemical Resource Engineering, College of Life Science and \\ Technology, Beijing University of Chemical Technology, Beijing, China
}

OPEN ACCESS

Edited by:

Ruixin Zhu,

Tongji University, China

Reviewed by:

Martin Bahls,

University Medicine Greifswald,

Germany

Daniela Carnevale

Sapienza University of Rome, Italy

*Correspondence:

Yan-Dong Wang

ydwangbuct2009@163.com

Wei-Dong Chen

wdchen666@163.com

Specialty section:

This article was submitted to Gastrointestinal and Hepatic

Pharmacology,

a section of the journal

Frontiers in Pharmacology

Received: 27 February 2017

Accepted: 10 April 2017

Published: 24 April 2017

Citation:

Lv X, Kong J, Chen W-D and

Wang Y-D (2017) The Role of the Apelin/APJ System in the Regulation

of Liver Disease.

Front. Pharmacol. 8:221.

doi: 10.3389/fphar.2017.00221
Apelin is an endogenous peptide that is a ligand for the APJ receptor (angiotensin II receptor like-1, AT-1). The apelin/APJ system is distributed in diverse periphery organ tissues. It has been shown that the apelin/APJ system plays various roles in physiology and pathophysiology of many organs. It regulates cardiovascular development or cardiac disease, glycometabolism and fat metabolism as well as metabolic disease. The apelin/APJ system participates in various cell activities such as proliferation, migration, apoptosis or inflammation. However, apelin/APJ function in the liver is still under investigation. In the liver, the apelin-APJ system could play an inhibitory role in liver regeneration and promote Fas-induced apoptosis. It may participate in the formation of hepatic fibrosis or cirrhosis, and even cancer. In this review, we summarize the role of the apelin/APJ system in liver disease.

Keywords: Apelin, APJ, liver disease, peptide, angiotensin II receptor like-1

\section{INTRODUCTION}

As an endogenous peptide, apelin acts as a ligand for the APJ receptor (angiotensin II receptor like-1, AT-1). Apelin is a 77 amino acid preproprotein and its isoforms include apelin-13, -16, -17 and -36 . Each isoform has distinct activity and the shorter isoform is a more potent activator for APJ. Both apelin-13 and apelin-17 have much stronger activity than apelin-36 (Kawamata et al., 2001; De Mota et al., 2004; Chaves-Almagro et al., 2015). The receptor binding affinities of apelin-13 and apelin-36 are different, which causes different intracellular signaling of APJ. APJ is composed of 377 amino acids. It has a seven transmembrane domain and is a $\mathrm{G}$ protein-coupled receptor. It is believed that apelin is the only endogenous ligand for APJ (Simpkin et al., 2007; Lee et al., 2010; Chapman et al., 2014).

It has been demonstrated that the apelin/APJ system plays important and various roles in the physiology and pathophysiology of many organs, including regulation of blood pressure, cardiac contractility, angiogenesis, metabolic balance, and cell proliferation, apoptosis or inflammation. However, whether apelin/APJ has important functions in liver disease is still under investigation. Liver disease includes liver fibrosis or cirrhosis, viral hepatitis, hepatocellular carcinoma (HCC), alcoholic fatty liver (ALD) as well as non-alcoholic fatty liver disease (NALD). In this review, we summarize the latest studies on the functions of the apelin/APJ system in liver disease.

\section{DISTRIBUTION AND EXPRESSION OF APELIN IN LIVER}

Apelin is expressed in the heart, endothelium, vascular smooth muscle cells (VSMCs), brain, kidney, testis, ovary, liver and adipose tissue, with the highest expression levels in the lung and 
the mammary gland (Hosoya et al., 2000; Kawamata et al., 2001). Chu et al. (2013) reported that APJ is expressed in primary hepatocytes, liver tissues of mouse and HepG2 cells, but the expression levels of apelin were lower than that of APJ in liver. The study of Jeong et al. (2014) showed that apelin expression was decreased by silencing the activating transcription factor 4 (ATF4) in HepG2 cells. Principe et al. (2008) reported that the apelin/APJ expression was increased sharply in the hepatic tissue of cirrhotic rats, and APJ expression was 300 times more than that of the control groups. In comparison with the control, the circulating levels of apelin were markedly increased in rats with cirrhosis. It has been found that APJ was expressed in Kupffer cells, and it inhibited liver regeneration after partial hepatectomy in mice (Yoshiya et al., 2015). In addition, the apelin/APJ was expressed in human hepatic stellate cells (HSCs), and promoted liver fibrosis or cirrhosis progression (Melgar-Lesmes et al., 2010; Yokomori et al., 2011).

\section{THE DOWNSTREAM AND UPSTREAM FACTORS OF APELIN/APJ}

It has been shown that apelin/APJ signaling is coupled to pertussis toxin (PTX)-sensitive $\mathrm{G}$ proteins and activates protein kinase C (PKC) in cultured cells (Masri et al., 2002) or mouse tissues (Liu et al., 2015). Both apelin-36 and apelin-13 can activate the same intracellular effectors. However, there are differences in their desensitization patterns and coupled G-proteins (Masri et al., 2006). The studies have demonstrated that apelin binds to APJ, which leads to the phosphorylation of protein kinase B (Akt) and extracellular signal-regulated kinase (ERK) in many cell proliferation or migration activities. The Akt signaling pathway has been shown to contribute to cell migration. Also, the ERK/Akt-p70S6K pathway regulates cell proliferation (Masri et al., 2004; Liu et al., 2010; Langelaan et al., 2013). It has been recently shown that a heterodimer is formed with APJ and $\mathrm{K}$-opioid receptors and this leads to the phosphorylation of ERK, resulting in increasing cell proliferation ( $\mathrm{Li}$ et al., 2012; Chaves-Almagro et al., 2015). Moreover, APJ/apelin system induces intercellular adhesion molecule-1 (ICAM-1) expression via the NF- $\mathrm{KB} / \mathrm{c}$-Jun $\mathrm{N}$-terminal kinase (JNK) signal pathway (Lu et al., 2012). Yasuzaki et al. (2013) has demonstrated that apelin/APJ signaling may promote Fas-induced liver injury via the phosphorylation of JNK in mice administered by intraperitoneal injection of an agonistic anti-Fas antibody (Jo2) (Yasuzaki et al., 2013). It was also shown that after partial hepatectomy and being given exogenous F13A, which is a specific APJ antagonist, liver regeneration was increased by enhancement of STAT3 and ERK1/2, but not phosphatidylinositol 3-kinase (PI3K)/Akt signaling. These results suggest that the apelin/APJ inhibits hepatocyte proliferation after partial hepatectomy in mice. Also, apelin was shown to possibly inhibit cell apoptosis in osteoblasts. Anti-apoptosis is mediated by the induction of Bcl2 protein expression and activation of the PI3K/Akt signaling pathway (Xie et al., 2007; Alastalo et al., 2011) (Figure 1).

The studies have reported that insulin, TNF- $\alpha$, all-transretinoic acid (ATRA) and hypoxic conditions may increase apelin expression in vivo and in vitro, and some transcription factors may regulate apelin gene transcription or expression. However, in different tissues or cells, apelin/APJ expression has different levels with the same stimulant. For example, Daviaud et al. (2006) reported that TNF- $\alpha$ up-regulated apelin expression in human and mouse adipose tissue while Melgar-Lesmes et al. $(2010,2011)$ revealed that, in HSCs, TNF- $\alpha$ decreased apelin/APJ expression at mRNA and protein levels, but increased apelin/APJ expression in HepG2 cells. Overexpression of Stat 3 and upstream stimulatory factor $1 / 2$ may increase apelin promoter transcription activity (Wang et al., 2006; Han et al., 2008). Increased apelin/APJ in liver tissues has been shown to occur with hypoxia (MelgarLesmes et al., 2011). Under hypoxic conditions, hypoxia inducible factor- $1 \alpha$ (HIF-1 $\alpha$ ) binds to the first intron of the apelin gene and the hypoxia-responsive element in the apelin promoter. It then increases apelin expression (Ronkainen et al., 2007; Eyries et al., 2008). The studies have identified that apelin promoter transcriptional activity increased through endothelial myocyte enhancer factor 2 (MEF2) transcription factors in the developing cardiovascular system (Kang et al., 2013), but its activity was inhibited by ATF4 in HepG2 cells (Jeong et al., 2014). The molecular mechanisms of the regulation of APJ gene transcription, however, are still unknown. The other investigations have also shown that both APJ and apelin genes are probably regulated by specificity protein 1 (Sp1) (O'Carroll et al., 2006; Hata et al., 2007). Our previous study showed that Sp1 and RAR $\alpha$ formed a transcriptional activation complex, leading to an up-regulation of apelin expression in rat VSMCs (Lv et al., 2013).

\section{FUNCTION OF THE APELIN/APJ IN PHYSIOLOGY AND PATHOPHYSIOLOGY OF THE LIVER}

In the liver, the apelin/APJ system could play an inhibitory role in liver regeneration and promote Fas-induced apoptosis. It may participate in formation of hepatic fibrosis or cirrhosis, and even cancer. The expression of apelin/APJ and related liver disease has been shown in Table 1.

Apelin/APJ signaling may promote Fas-induced liver injury via JNK activation. In the $\mathrm{APJ}^{-/-}$mice, it had been demonstrated that liver apoptosis and injury were significantly alleviated compared with that in wild-type mice, and JNK activation was strongly inhibited (Yasuzaki et al., 2013). F13A, a specific APJ antagonist, could directly promote the activation of Kupffer cells and enhance the secretion of TNF- $\alpha$ and IL-6, which could increase hepatocyte proliferation with partial resection of the liver. However, this effect has not arisen in hepatocytes or hepatic stellate cells. Moreover, the evidence revealed that the relationship between apelin and TNF- $\alpha$ was important in the development of liver regeneration (Fausto et al., 2012). Sagiroglu et al. (2014) reported that exogenous apelin administration as pharmacological preconditioning alleviated hepatic ischemia reperfusion (I/R) injury in rats. These findings suggest that apelin/APJ system is involved in the process of liver regeneration.

The study showed that apelin was associated with the development of liver fibrosis. The in vivo study revealed 


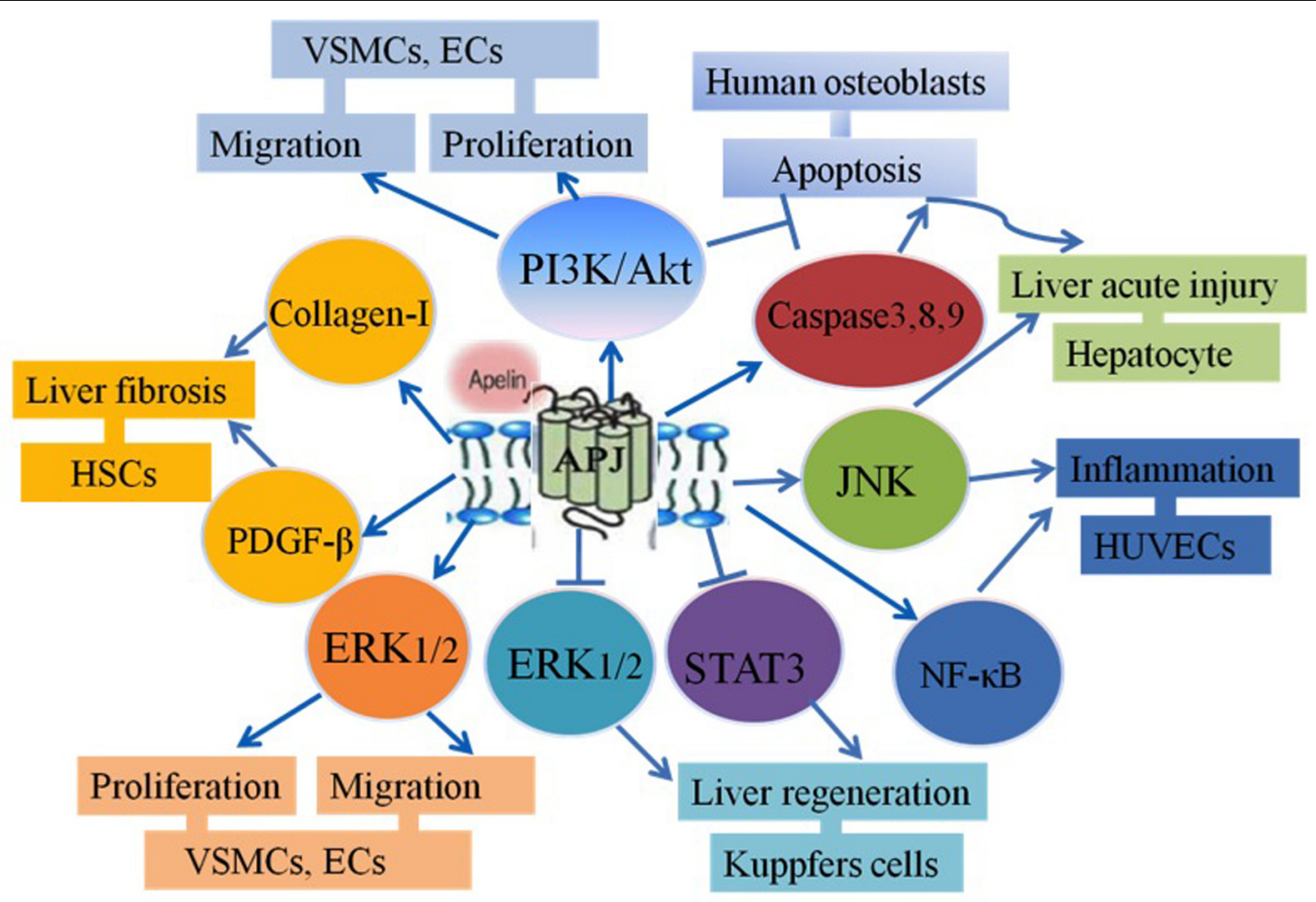

FIGURE 1 | The functions of apelin/APJ in liver disease.

TABLE 1 | Expression of the apelin/APJ system in patients, tumor tissues, or cell lines.

\begin{tabular}{|c|c|c|c|c|c|}
\hline Liver disease type & Patients/tissues/cells & mRNA & Protein & Serum levels & Reference \\
\hline Hepatocellular carcinoma & Liver of hepatocellular carcinoma patients & Apelin $\uparrow$ & - & - & Muto et al., 2014 \\
\hline Non-alcoholic fatty liver disease & Human serum & - & - & Apelin $\uparrow$ & Aktas et al., 2011 \\
\hline Liver cirrhosis & Human serum, Rat and human tissues & Apelin $\uparrow \mathrm{APJ} \uparrow$ & Apelin $\uparrow \mathrm{APJ} \uparrow$ & Apelin $\uparrow$ & $\begin{array}{l}\text { Principe et al., 2008; } \\
\text { Melgar-Lesmes et al., 2010, } \\
\text { 2011; Yokomori et al., 2011; } \\
\text { Lim et al., } 2016\end{array}$ \\
\hline Fas-induced liver injury & Mouse tissues & $\mathrm{APJ} \uparrow$ & APJ $\uparrow$ & - & Yasuzaki et al., 2013 \\
\hline Hepatitis C & Patients HSCs, hepatocytes & Apelin $\uparrow$ & Apelin $\uparrow$ & Apelin $\uparrow$ & $\begin{array}{l}\text { El-Mesallamy et al., 2011; } \\
\text { Farid et al., } 2014\end{array}$ \\
\hline
\end{tabular}

that apelin/APJ could accelerate fibrosis progression in $\mathrm{CCL}_{4}{ }^{-}$ treated rats (Reichenbach et al., 2012). Melgar-Lesmes et al. (2011) reported that apelin expression in HSCs was enhanced under hypoxic or proinflammatory conditions, and apelin could increase the synthesis of platelet-derived growth factor $\beta$ receptor (PDGF- $\beta$ ) and collagen-I in LX-2 cells. Yokomori et al. (2011) reported that, in cultured HSCs, APJ expression was sharply increased by PDGF- $\beta$. The function of apelin/APJ in liver fibrosis still needs to be investigated.

A recent clinical investigation reported that serum apelin was associated with histological and hemodynamic states of chronic liver disease (Lim et al., 2016). Drougard et al. (2014) reported that hypothalamic apelin regulated hepatic glucose metabolism in mice fed a high-fat diet. Moreover, in human NALD, plasma apelin-12 levels were higher than that in healthy individuals (Ercin et al., 2010). The levels of apelin were also positively correlated with the homeostasis model assessment (HOMA) index and body mass index (BMI) in liver metabolic disease.

Recent studies have also reported that the apelin/APJ system participates in many kinds of cancer including lung, gastroesophageal, colonic, prostate and endometrial cancer (Picault et al., 2014; Altinkaya et al., 2015; Lv et al., 2016). The functions of apelin in liver cancer are still not clear. Apelin expression was upregulated in F26/KMUH cancer-associated 
fibroblast (CAFs) in HCC. Also, the higher levels of apelin/APJ expression were found in HCC based on clinical specimens. Tumor growth was obviously inhibited after blocking apelin/APJ signaling with exogenous F13A in a HCC subcutaneous mouse tumor model, compared to the control group (Muto et al., 2014). These results suggest that apelin/APJ is associated with liver cancer but the roles of apelin/APJ in liver cancer need to be further investigated.

\section{CONCLUSION}

Recent studies have reported there are multiple roles for the apelin/APJ system in liver disease, including acute liver injury, liver regeneration, formation of cirrhotic liver and fibrosis progression. Apelin/APJ has unique functions as a regulator of cell proliferation, apoptosis, pro-inflammatory activity and revascularization. In addition, apelin/APJ gene expression is temporally increased during liver cirrhotic development and it decreased in stabilized liver fibrosis formation. The validation of using apelin/APJ as a biomarker in different liver diseases would also be a crucial step toward its clinical use. Also, further experimental or clinical findings will help to determine the potential of therapeutic strategies targeting the apelin/APJ system for treatment of liver disease.

\section{REFERENCES}

Aktas, B., Yilmaz, Y., Eren, F., Yonal, O., Kurt, R., Alahdab, Y. O., et al. (2011). Serum levels of vaspin, obestatin, and apelin-36 in patients with nonalcoholic fatty liver disease. Metabolism 60, 544-549. doi: 10.1016/j.metabol.2010.05.008

Alastalo, T. P., Li, M., Perez Vde, J., Pham, D., Sawada, H., Wang, J. K., et al. (2011). Disruption of PPARgamma/beta-catenin-mediated regulation of apelin impairs BMP-induced mouse and human pulmonary arterial EC survival. J. Clin. Invest. 121, 3735-3746. doi: 10.1172/JCI43382

Altinkaya, S. O., Nergiz, S., Kucuk, M., and Yuksel, H. (2015). Apelin levels are higher in obese patients with endometrial cancer. J. Obstet. Gynaecol. Res. 41, 294-300. doi: 10.1111/jog. 12503

Chapman, N. A., Dupre, D. J., and Rainey, J. K. (2014). The apelin receptor: physiology, pathology, cell signalling, and ligand modulation of a peptideactivated class A GPCR. Biochem. Cell Biol. 92, 431-440. doi: 10.1139/bcb-20140072

Chaves-Almagro, C., Castan-Laurell, I., Dray, C., Knauf, C., Valet, P., and Masri, B. (2015). Apelin receptors: from signaling to antidiabetic strategy. Eur. J. Pharmacol. 763, 149-159. doi: 10.1016/j.ejphar.2015.05.017

Chu, J., Zhang, H., Huang, X., Lin, Y., Shen, T., Chen, B., et al. (2013). Apelin ameliorates TNF-alpha-induced reduction of glycogen synthesis in the hepatocytes through G protein-coupled receptor APJ. PLoS ONE 8:e57231. doi: 10.1371/journal.pone.0057231

Daviaud, D., Boucher, J., Gesta, S., Dray, C., Guigne, C., Quilliot, D., et al. (2006). TNFalpha up-regulates apelin expression in human and mouse adipose tissue. FASEB J. 20, 1528-1530. doi: 10.1096/fj.05-5243fje

De Mota, N., Reaux-Le Goazigo, A., El Messari, S., Chartrel, N., Roesch, D., Dujardin, C., et al. (2004). Apelin, a potent diuretic neuropeptide counteracting vasopressin actions through inhibition of vasopressin neuron activity and vasopressin release. Proc. Natl. Acad. Sci. U.S.A. 101, 10464-10469. doi: 10.1073/pnas.0403518101

Drougard, A., Duparc, T., Brenachot, X., Carneiro, L., Gouaze, A., Fournel, A., et al. (2014). Hypothalamic apelin/reactive oxygen species signaling controls hepatic glucose metabolism in the onset of diabetes. Antioxid. Redox Signal. 20, 557-573. doi: 10.1089/ars.2013.5182

\section{AUTHOR CONTRIBUTIONS}

XL wrote the manuscript. JK helped to prepare figures. W-DC and Y-DW reviewed, edited and revised the manuscript.

\section{FUNDING}

This work is supported by the National Natural Science Foundation of China (Grant No. 81472232 and Grant No. 81270522) to W-DC, the National Natural Science Foundation of China (Grant No. 81370537 and Grant No. 81672433) to Y-DW, the National Natural Science Foundation of China (Grant No. U1404310) to XL, Program for Science \& Technology Innovation Talents in Universities of Henan Province (HASTIT, Grant No. 13HASTIT024) and Plan for Scientific Innovation Talent of Henan Province to W-DC and the Fundamental Research Funds for the Central Universities (Grant No. YS1407 and 2050205) to Y-DW.

\section{ACKNOWLEDGMENT}

We apologize to colleagues whose work could not be cited due to space limitations.

El-Mesallamy, H. O., Hamdy, N. M., Rizk, H. H., and El-Zayadi, A.-R. (2011). Apelin serum level in Egyptian patients with chronic hepatitis C. Mediators Inflamm. 2011:703031. doi: 10.1155/2011/703031

Ercin, C. N., Dogru, T., Tapan, S., Kara, M., Haymana, C., Karadurmus, N., et al. (2010). Plasma apelin levels in subjects with nonalcoholic fatty liver disease. Metabolism 59, 977-981. doi: 10.1016/j.metabol.2009.10.019

Eyries, M., Siegfried, G., Ciumas, M., Montagne, K., Agrapart, M., Lebrin, F., et al. (2008). Hypoxia-induced apelin expression regulates endothelial cell proliferation and regenerative angiogenesis. Circ. Res. 103, 432-440. doi: 10.1161/CIRCRESAHA.108.179333

Farid, R. M., Abu-Zeid, R. M., and El-Tawil, A. (2014). Emerging role of adipokine apelin in hepatic remodelling and initiation of carcinogensis in chronic hepatitis C patients. Int. J. Clin. Exp. Pathol. 7, 2707-2717.

Fausto, N., Campbell, J. S., and Riehle, K. J. (2012). Liver regeneration. J. Hepatol. 57, 692-694. doi: 10.1016/j.jhep.2012.04.016

Han, S., Wang, G., Qi, X., Englander, E. W., and Greeley, G. H. Jr. (2008). Involvement of a Stat3 binding site in inflammation-induced enteric apelin expression. Am. J. Physiol. Gastrointest. Liver Physiol. 295, G1068-G1078. doi: 10.1152/ajpgi.90493.2008

Hata, J., Matsuda, K., Ninomiya, T., Yonemoto, K., Matsushita, T., Ohnishi, Y., et al. (2007). Functional SNP in an Sp1-binding site of AGTRL1 gene is associated with susceptibility to brain infarction. Hum. Mol. Genet. 16, 630-639. doi: $10.1093 / \mathrm{hmg} / \mathrm{ddm} 005$

Hosoya, M., Kawamata, Y., Fukusumi, S., Fujii, R., Habata, Y., Hinuma, S., et al. (2000). Molecular and functional characteristics of APJ. Tissue distribution of mRNA and interaction with the endogenous ligand apelin. J. Biol. Chem. 275, 21061-21067. doi: 10.1074/jbc.M908417199

Jeong, K., Oh, Y., Kim, S. J., Kim, H., Park, K. C., Kim, S. S., et al. (2014). Apelin is transcriptionally regulated by ER stress-induced ATF4 expression via a p38 MAPK-dependent pathway. Apoptosis 19, 1399-1410. doi: 10.1007/s10495014-1013-0

Kalafateli, M., Triantos, C., Tsochatzis, E., Michalaki, M., Koutroumpakis, E., Thomopoulos, K., et al. (2015). Adipokines levels are associated with the severity of liver disease in patients with alcoholic cirrhosis. World J. Gastroenterol. 21, 3020-3029. doi: 10.3748/wjg.v21.i10.3020 
Kang, Y., Kim, J., Anderson, J. P., Wu, J., Gleim, S. R., Kundu, R. K., et al. (2013). Apelin-APJ signaling is a critical regulator of endothelial MEF2 activation in cardiovascular development. Circ. Res. 113, 22-31. doi: 10.1161/CIRCRESAHA. 113.301324

Kawamata, Y., Habata, Y., Fukusumi, S., Hosoya, M., Fujii, R., Hinuma, S., et al. (2001). Molecular properties of apelin: tissue distribution and receptor binding. Biochim. Biophys. Acta 1538, 162-171. doi: 10.1016/S0167-4889(00)00143-9

Langelaan, D. N., Reddy, T., Banks, A. W., Dellaire, G., Dupre, D. J., and Rainey, J. K. (2013). Structural features of the apelin receptor N-terminal tail and first transmembrane segment implicated in ligand binding and receptor trafficking. Biochim. Biophys. Acta 1828, 1471-1483. doi: 10.1016/j.bbamem.2013. 02.005

Lee, D. K., Ferguson, S. S., George, S. R., and O’Dowd, B. F. (2010). The fate of the internalized apelin receptor is determined by different isoforms of apelin mediating differential interaction with beta-arrestin. Biochem. Biophys. Res. Commun. 395, 185-189. doi: 10.1016/j.bbrc.2010.03.151

Li, Y., Chen, J., Bai, B., Du, H., Liu, Y., and Liu, H. (2012). Heterodimerization of human apelin and kappa opioid receptors: roles in signal transduction. Cell. Signal. 24, 991-1001. doi: 10.1016/j.cellsig.2011.12.012

Lim, Y. L., Choi, E., Jang, Y. O., Cho, Y. Z., Kang, Y. S., Baik, S. K., et al. (2016). Clinical implications of the serum apelin level on portal hypertension and prognosis of liver cirrhosis. Gut Liver 10, 109-116. doi: 10.5009/gnl14345

Liu, C., Su, T., Li, F., Li, L., Qin, X., Pan, W., et al. (2010). PI3K/Akt signaling transduction pathway is involved in rat vascular smooth muscle cell proliferation induced by apelin-13. Acta Biochim. Biophys. Sin. 42, 396-402. doi: $10.1093 /$ abbs/gmq035

Liu, Q., Hu, T., He, L., Huang, X., Tian, X., Zhang, H., et al. (2015). Genetic targeting of sprouting angiogenesis using Apln-CreER. Nat. Commun. 6:6020. doi: $10.1038 /$ ncomms 7020

Lu, Y., Zhu, X., Liang, G. X., Cui, R. R., Liu, Y., Wu, S. S., et al. (2012). ApelinAPJ induces ICAM-1, VCAM-1 and MCP-1 expression via NF-KB/JNK signal pathway in human umbilical vein endothelial cells. Amino Acids 43, 2125-2136. doi: 10.1007/s00726-012-1298-7

Lv, D., Li, L., Lu, Q., Li, Y., Xie, F., Li, H., et al. (2016). PAK1-cofilin phosphorylation mediates human lung adenocarcinoma cells migration induced by apelin-13. Clin. Exp. Pharm. Physiol. 43, 569-579. doi: 10.1111/1440-1681.12563

Lv, X. R., Zheng, B., Li, S. Y., Han, A. L., Wang, C., Shi, J. H., et al. (2013). Synthetic retinoid Am80 up-regulates apelin expression by promoting interaction of RARalpha with KLF5 and Sp1 in vascular smooth muscle cells. Biochem. J. 456, 35-46. doi: 10.1042/BJ20130418

Masri, B., Lahlou, H., Mazarguil, H., Knibiehler, B., and Audigier, Y. (2002). Apelin (65-77) activates extracellular signal-regulated kinases via a PTX-sensitive G protein. Biochem. Biophys. Res. Commun. 290, 539-545. doi: 10.1006/bbrc.2001. 6230

Masri, B., Morin, N., Cornu, M., Knibiehler, B., and Audigier, Y. (2004). Apelin (65-77) activates p70 S6 kinase and is mitogenic for umbilical endothelial cells. FASEB J. 18, 1909-1911. doi: 10.1096/fj.04-1930fje

Masri, B., Morin, N., Pedebernade, L., Knibiehler, B., and Audigier, Y. (2006). The apelin receptor is coupled to Gi1 or Gi2 protein and is differentially desensitized by apelin fragments. J. Biol. Chem. 281, 18317-18326. doi: 10.1074/ jbc.M600606200

Melgar-Lesmes, P., Casals, G., Pauta, M., Ros, J., Reichenbach, V., Bataller, R., et al. (2010). Apelin mediates the induction of profibrogenic genes in human hepatic stellate cells. Endocrinology 151, 5306-5314. doi: 10.1210/en.2010-0754

Melgar-Lesmes, P., Pauta, M., Reichenbach, V., Casals, G., Ros, J., Bataller, R., et al. (2011). Hypoxia and proinflammatory factors upregulate apelin receptor expression in human stellate cells and hepatocytes. Gut 60, 1404-1411. doi: $10.1136 /$ gut.2010.234690
Muto, J., Shirabe, K., Yoshizumi, T., Ikegami, T., Aishima, S., Ishigami, K., et al. (2014). The apelin-APJ system induces tumor arteriogenesis in hepatocellular carcinoma. Anticancer Res. 34, 5313-5320.

O'Carroll, A. M., Lolait, S. J., and Howell, G. M. (2006). Transcriptional regulation of the rat apelin receptor gene: promoter cloning and identification of an Sp1 site necessary for promoter activity. J. Mol. Endocrinol. 36, 221-235. doi: 10.1677/jme.1.01927

Picault, F. X., Chaves-Almagro, C., Projetti, F., Prats, H., Masri, B., and Audigier, Y. (2014). Tumour co-expression of apelin and its receptor is the basis of an autocrine loop involved in the growth of colon adenocarcinomas. Eur. J. Cancer 50, 663-674. doi: 10.1016/j.ejca.2013.11.017

Principe, A., Melgar-Lesmes, P., Fernandez-Varo, G., del Arbol, L. R., Ros, J., Morales-Ruiz, M., et al. (2008). The hepatic apelin system: a new therapeutic target for liver disease. Hepatology 48, 1193-1201. doi: 10.1002/hep.22467

Reichenbach, V., Ros, J., Fernandez-Varo, G., Casals, G., Melgar-Lesmes, P., Campos, T., et al. (2012). Prevention of fibrosis progression in CCl4-treated rats: role of the hepatic endocannabinoid and apelin systems. J. Pharmacol. Exp. Ther. 340, 629-637. doi: 10.1124/jpet.111.188078

Ronkainen, V. P., Ronkainen, J. J., Hanninen, S. L., Leskinen, H., Ruas, J. L., Pereira, T., et al. (2007). Hypoxia inducible factor regulates the cardiac expression and secretion of apelin. FASEB J. 21, 1821-1830. doi: 10.1096/fj.067294com

Sagiroglu, T., Aksoy, M. B., Sagiroglu, G., Tozkir, H., Oguz, S., Yalta, T., et al. (2014). Effect of leptin and apelin preconditioning on hepatic ischemia reperfusion injury in rats. Indian J. Surg. 76, 111-116. doi: 10.1007/s12262-012-0676-4

Simpkin, J. C., Yellon, D. M., Davidson, S. M., Lim, S. Y., Wynne, A. M., and Smith, C. C. (2007). Apelin-13 and apelin-36 exhibit direct cardioprotective activity against ischemia-reperfusion injury. Basic Res. Cardiol. 102, 518-528. doi: 10.1007/s00395-007-0671-2

Wang, G., Qi, X., Wei, W., Englander, E. W., and Greeley, G. H. Jr. (2006). Characterization of the 5'-regulatory regions of the rat and human apelin genes and regulation of breast apelin by USF. FASEB J. 20, 2639-2641. doi: 10.1096/fj. 06-6315fje

Xie, H., Yuan, L. Q., Luo, X. H., Huang, J., Cui, R. R., Guo, L. J., et al. (2007). Apelin suppresses apoptosis of human osteoblasts. Apoptosis 12, 247-254. doi: 10.1007/s10495-006-0489-7

Yasuzaki, H., Yoshida, S., Hashimoto, T., Shibata, W., Inamori, M., Toya, Y., et al. (2013). Involvement of the apelin receptor APJ in Fas-induced liver injury. Liver Int. 33, 118-126. doi: 10.1111/liv.12006

Yokomori, H., Oda, M., Yoshimura, K., Machida, S., Kaneko, F., and Hibi, T. (2011). Overexpression of apelin receptor (APJ/AGTRL1) on hepatic stellate cells and sinusoidal angiogenesis in human cirrhotic liver. J. Gastroenterol. 46, 222-231. doi: 10.1007/s00535-010-0296-3

Yoshiya, S., Shirabe, K., Imai, D., Toshima, T., Yamashita, Y., Ikegami, T., et al. (2015). Blockade of the apelin-APJ system promotes mouse liver regeneration by activating Kupffer cells after partial hepatectomy. J. Gastroenterol. 50, 573-582. doi: 10.1007/s00535-014-0992-5

Conflict of Interest Statement: The authors declare that the research was conducted in the absence of any commercial or financial relationships that could be construed as a potential conflict of interest.

Copyright (c) 2017 Lv, Kong, Chen and Wang. This is an open-access article distributed under the terms of the Creative Commons Attribution License (CC BY). The use, distribution or reproduction in other forums is permitted, provided the original author(s) or licensor are credited and that the original publication in this journal is cited, in accordance with accepted academic practice. No use, distribution or reproduction is permitted which does not comply with these terms. 